\title{
Atividade antifúngica e proteção do tomateiro por extratos de plantas medicinais
}

\author{
Adriana T. Itako ${ }^{1}$, Kátia R. F. Schwan-Estrada ${ }^{1}$, João B. Tolentino Júnior ${ }^{1}$, José R. Stangarlin² \& Maria E. \\ da Silva Cruz ${ }^{1}$
}

${ }^{1}$ Departamento de Agronomia, Universidade Estadual de Maringá, 87020-900, Maringá, PR, Brasil; ${ }^{2}$ Centro de Ciências Agrárias, Universidade Estadual do Oeste do Paraná - UNIOESTE, 85960-000, Marechal Cândido Rondon, PR, Brasil

Autor para correspondência: Adriana Terumi Itako, e-mail: atitako@yahoo.com.br

\begin{abstract}
RESUMO
Este trabalho teve como objetivos avaliar, in vitro, a fungitoxicidade dos extratos brutos aquosos (EBAs) de Achillea millefolium, Artemisia camphorata, Cymbopogon citratus e Rosmarinus officinalis contra Alternaria solani e o efeito protetor destes extratos em plantas de tomateiro em casa-de-vegetação. Para verificar a atividade antifúngica, os EBAs foram incorporados ao BDA e avaliadas a inibição do crescimento micelial, a esporulação e a germinação de conídios. O efeito protetor em plantas foi avaliado através da pulverização preventiva ( $72 \mathrm{~h}$ antes da inoculação) dos EBAs, nas concentrações de 10 e 20\%, nas duas primeiras folhas. A severidade da doença foi verificada 15 dias após a inoculação. Verificou-se que os EBAs não inibiram o crescimento micelial, mas tiveram efeitos significativos na redução da esporulação e da germinação de conídios, principalmente os EBAs de A. camphorata, C. citratus e R. officinalis, a partir da concentração de $20 \%$. Já em relação à proteção das plantas verificou-se uma redução no número de lesões em relação à testemunha, nas folhas acima das tratadas, observando o efeito sistêmico dos extratos. Os extratos estudados podem ser promissores no controle da pinta-preta em tomateiro.
\end{abstract}

Palavras-chave: Alternaria solani, controle alternativo.

\begin{abstract}
Antifungal activity and protection of tomato plants by extracts of medicinal plants

The present work aimed to evaluate, in vitro, the fungitoxicity of aqueous crude extracts (ACEs) of Achillea millefolium, Artemisia camphorata, Cymbopogon citratus and Rosmarinus officinalis to Alternaria solani and their protective effect on tomato plants under greenhouse conditions. To evaluate the antifungal activity of ACEs, they were incorporated into potato-dextrose-agar medium and the inhibition of mycelial growth, sporulation and conidia germination was evaluated. The protective effect in plants was evaluated by means of preventive spraying ( 72 hours before inoculation) with ACE, at concentrations of 10 and 20\%, in the first two leaves. Disease severity was verified 15 days after inoculation. It was observed that ACEs did not inhibit mycelial growth, but they had significant effects on the reduction of germination and sporulation, especially the ACEs of A. camphorata, C. citratus and R. officinalis, at concentrations up to $20 \%$. A reduction was observed in the number of lesions in relation to the control, in leaves above those treated, observing the systemic effect of the extracts. The extracts from the studied plants may be promising in the control of early blight on tomato plants.
\end{abstract}

Keywords: Alternaria solani, alternative control.

A pinta preta, causada por Alternaria solani Sorauer é uma das mais importantes e freqüentes doenças fúngicas da cultura do tomateiro no Brasil. Apresenta alto potencial destrutivo incidindo sobre folhas, hastes, pecíolos e frutos, ocasionando elevados prejuízos econômicos (Kurozawa \& Pavan, 2005). Atualmente, as medidas de controle se baseiam no uso de produtos químicos, pois as variedades tradicionalmente cultivadas são suscetíveis ao patógeno (Vale et al., 2000). Em estudo para diagnosticar o uso de produtos químicos, dentre 18 importantes culturas da agricultura paulista, o tomateiro representou uma das culturas mais problemáticas quanto ao uso de agrotóxicos, registrando um elevado percentual de intoxicações em trabalhadores (Vicente et al., 2002).

Pesquisas desenvolvidas com extrato bruto ou óleo essencial, obtidos de plantas medicinais, têm indicado o potencial das mesmas no controle de fitopatógenos, tanto por sua ação fungitóxica direta quanto por alterações fisiológicas na planta, como indução de enzimas relacionadas à patogênese e fitolexinas, lignificação da folha, entre outras (Stangarlin et al., 1999; Schwan-Estrada \& Stangarlin, 2005).

Exemplos de controle de doenças de plantas com extrato vegetais incluem os seguintes tratamentos: o extrato de Cymbopogon citratus a 10\% inibiu completamente o crescimento in vitro de vários patógenos causadores de podridão radicular em feijoeiro (Valarini et al., 1994); o controle da mancha marrom (Bipolaris sorokiniana) em trigo usando extrato de Artemisia camphorata (Franzener et al., 2003); da pinta preta (A. solani) em tomateiro por extrato 
de Curcuma longa (Balbi-Peña et al.,2006b); o controle do mofo branco (Sclerotinia sclerotiorum) em alface por extrato de Zingiber officinale (Rodrigues et al., 2007); e o controle de Xanthomonas axonopodis pv. manihotis em mandioca por extrato de Curcuma longa (Kuhn et al., 2006).

$\mathrm{O}$ objetivo deste trabalho foi verificar in vitro o efeito de extrato bruto aquoso (EBA) das plantas medicinais Achillea millefolium (mil-folhas), Artemisia camphorata (cânfora), Cymbopogon citratus (capim-limão) e Rosmarinus officinalis (alecrim) no crescimento micelial, germinação e esporulação de conídios de Alternaria solani, bem como o efeito protetor destes extratos em plantas de tomateiro,em casa-de-vegetação.

Folhas frescas e sadias das plantas medicinais nas concentrações 1, 10, 20 e $40 \%$ (g peso fresco/mL de caldo de batata) foram trituradas, separadamente, em caldo de batata por $3 \mathrm{~min}$ em liquidificador. Os homogenatos resultantes foram filtrados em gaze e papel de filtro Whatman oㅜ․ Foi incorporado ágar ao meio e após a autoclavagem o meio foi distribuído em placas de Petri. Após a solidificação, um disco de $8 \mathrm{~mm}$ de diâmetro do micélio de $A$. solani, com 10 dias de idade, foi repicado para o centro de cada placa, as quais foram vedadas com filme plástico e mantidas a $24 \pm$ $2^{\circ} \mathrm{C}$ no escuro.

A avaliação do crescimento micelial foi realizada através de medições diárias do diâmetro das colônias, obtida pela média de duas medidas diametralmente opostas, e perdurou até o momento em que as colônias fúngicas no tratamento controle cobriram $2 / 3$ da superfície do meio de cultura. Nesse momento, também se realizou a contagem de conídios. Para isso, foram adicionados $10 \mathrm{~mL}$ de água destilada em cada placa, a suspensão foi filtrada em gaze e a contagem realizada em câmara de Neubauer.

No ensaio de germinação de conídios, alíquotas de $80 \mu \mathrm{l}$ da suspensão de conídios foram colocadas em pocinhos de placa utilizada em teste de ELISA e incubadas sob luz constante à temperatura de $24^{\circ} \mathrm{C}$ por um período de $3 \mathrm{~h}$. A germinação foi paralisada com $20 \mu \mathrm{l}$ do corante azul de algodão. A avaliação foi realizada pela contagem de conídios germinados, sendo considerados germinados quando apresentaram qualquer emissão de tubo germinativo.

Utilizou-se o delineamento experimental inteiramente casualizado com 17 tratamentos e quatro repetições, em esquema fatorial $4 \times 4+1$, cujos fatores foram 4 EBAs, 4 concentrações $(1 \%, 10 \%, 20 \%$ e $40 \%)$ e testemunha.

Para se avaliar a severidade da doença, sementes de tomate cv. Santa Clara foram semeadas em bandejas de isopor de 128 células. Aos 20 dias após a semeadura, as mudas foram transplantadas para vasos de $2 \mathrm{~L}$ contendo solo esterilizado e areia. O primeiro par de folhas foi pulverizado com EBA das plantas medicinais nas concentrações de $10 \% \mathrm{e}$ $20 \%$ até o ponto de escorrimento. Na testemunha aplicou-se água. Setenta e duas horas após a aplicação dos tratamentos, o primeiro par de folhas (tratadas) e o segundo par de folhas (não-tratadas) foram inoculadas com suspensão de conídios de $A$. solani $\left(10^{4}\right.$ conídios $\left./ \mathrm{mL}\right)$ e as plantas mantidas em sala de incubação a $25^{\circ} \mathrm{C}$ e condições de alta umidade relativa por 24h. A avaliação da severidade da doença foi realizada aos 15 dias após a inoculação, pela contagem do número de lesões nas folhas tratadas e não-tratadas. O delineamento experimental utilizado foi inteiramente casualizado, com três repetições, sendo cada parcela constituída por um vaso e duas plantas em cada vaso.

Em relação ao crescimento micelial de A. solani, observou-se que o mesmo foi igual ou superior à testemunha nos EBAs das plantas testadas, indicando que estes extratos não impediram o crescimento do fungo in vitro. Em relação à porcentagem de esporulação do fungo (Figura 1), podese verificar que o efeito dos EBAs na produção de conídios foi diferenciado quando os extratos foram utilizados em concentrações mais altas. Pela análise de regressão, o modelo ajustado foi exponencial, exceto para o EBA de $A$. millefolium, para qual nenhum modelo foi ajustado. Para os extratos de $A$. camphorata e de $C$. citratus nas concentrações de $20 \%$ e $40 \%$, a porcentagem de redução de esporulação foi de 91,$5 ; 98,4 ; 93,2$ e 99,0\%, respectivamente. Verificou-se que, com aumento na concentração do EBA (de $20 \%$ para $40 \%$ ), o incremento na porcentagem de inibição foi pequeno, observando-se que não há necessidade de concentrações tão altas para se obter efeitos satisfatórios. Para o extrato de $R$. officinalis, a partir da concentração de $10 \%$ já se verificou redução de $78,9 \%$ na esporulação.

$\mathrm{Na}$ análise de regressão da porcentagem de germinação de conídios (Figura 2), o modelo ajustado foi o linear para todos os tipos de extratos utilizados. Utilizando-se o extrato de $A$. camphorata a porcentagem de germinação foi de $79 \%$ a partir da concentração de $1 \%$ e $21,5 \%$ na concentração de $40 \%$. Para os extratos de C. citratus e R. officinalis a $20 \%$ e $40 \%$ os resultados obtidos foram: 63,3 e 39,$1 ; 59,5$ e $38,5 \%$, de germinação, respectivamente. Já para o extrato de $A$. millefolium o maior valor alcançado foi na concentração de $40 \%$ com germinação de $76 \%$, verificando-se que este extrato foi o que menos inibiu a germinação dos conídios de $A$. solani. Resultados semelhantes foram encontrados por Fiori et al. (2000) que verificaram que extratos de Eucalyptus citriodora, Ageratum conyzoides e A. millefollium além de inibirem o crescimento micelial, inibiram a germinação dos esporos de Didymella brioniae. Rodrigues et al. (2006) mostraram também a eficiência dos extratos de $Z$. officinalis e Corymbia citriodora na redução do crescimento micelial e produção de esporos de Helminthosporium sp. A atividade antifúngica de $A$. camphorata também foi verificada por Franzener et al. (2003), sendo que o extrato aquoso de $50 \%$ causou a inibição de $39 \%$ do crescimento micelial de $B$. sorokiniana, e a concentração de $10 \%$ já inibiu completamente a esporulação. Especificamente para $A$. solani, o trabalho de Balbi-Peña et al. (2006a) indicou que os extratos não autoclavados de Curcuma longa a $10 \%$ e $15 \%$ inibiram o crescimento micelial em 38,2 e $23,2 \%$, respectivamente, e a esporulação em 71,7 e $87 \%$, respectivamente. 



A

B

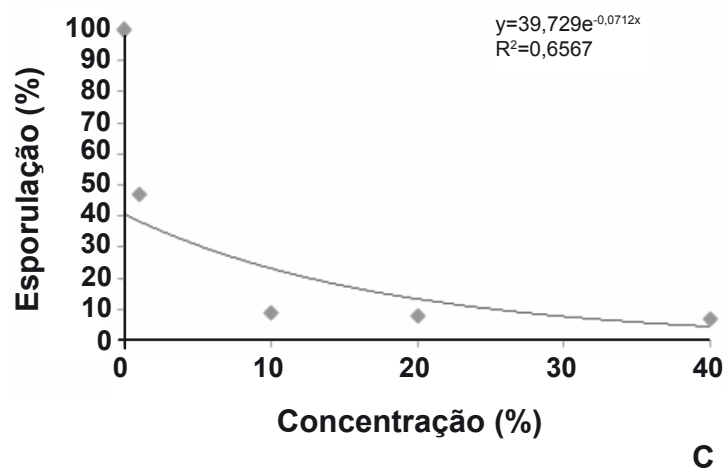

FIG. 1 - Esporulação (\%) de Alternaria solani sob diferentes concentrações de extratos brutos aquosos de (A) Artemisia camphorata (B) Cymbopogon citratus e (C) Rosmarinus officinalis.
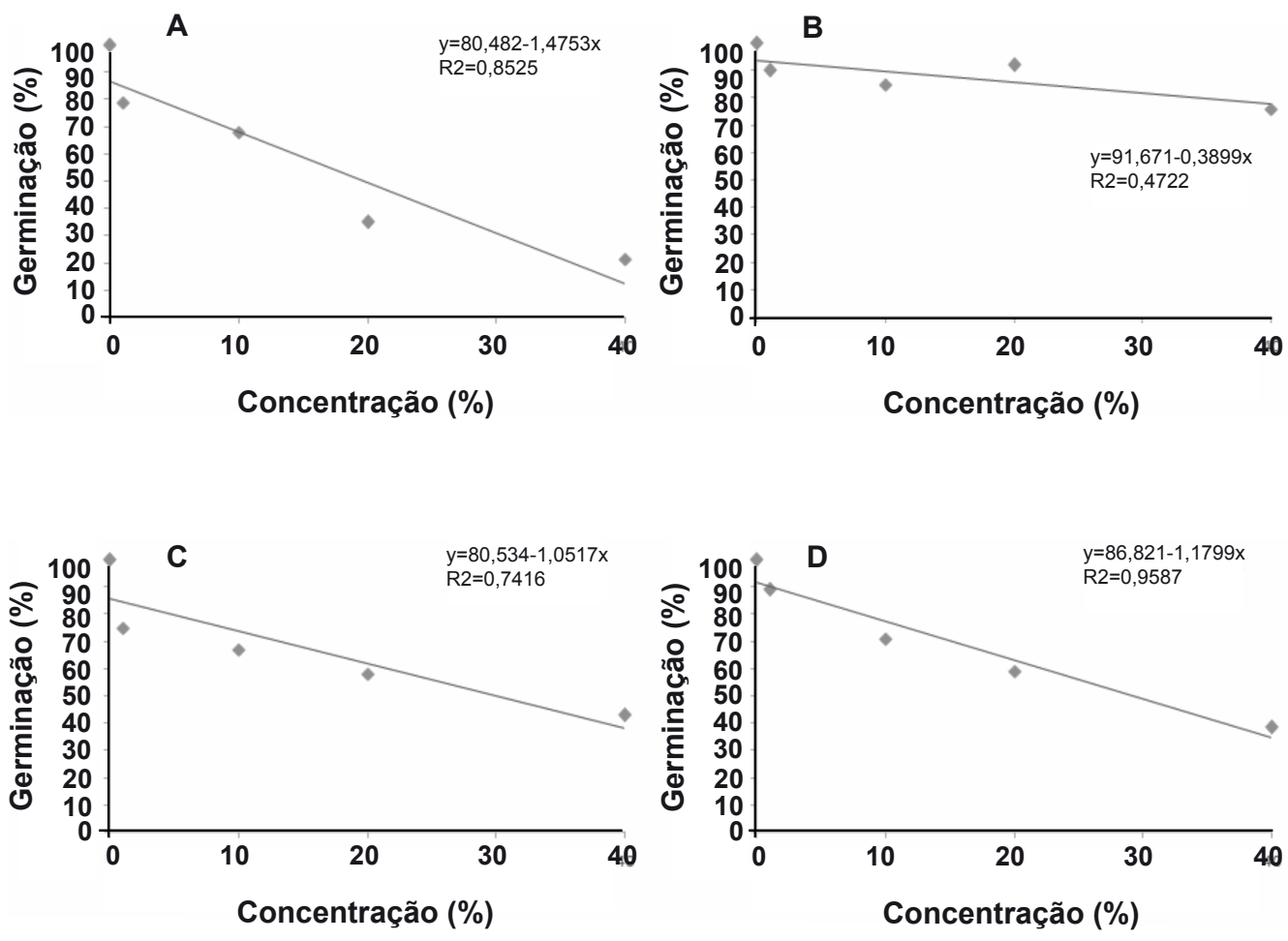

FIG. 2 - Germinação (\%) de conídios de Alternaria solani sob diferentes concentrações de extratos brutos e A. Artemisia camphorata; B. Achillea millefolium; C. Cymbopogon citratus e D. Rosmarinus offinalis. 
O número de lesões no primeiro par de folhas não diferiu estatisticamente da testemunha, observando que não houve proteção local (Tabela 1). Porém, no segundo par de folhas, que não recebeu tratamento algum, todos os EBAs utilizados diferiram estatisticamente da testemunha, apresentando número de lesões inferior. Com isso, pode-se verificar uma translocação sistêmica dos extratos. MontesBelmont \& Garcia-Licona (1997) trabalhando com o mesmo patossistema, observaram que a infusão de folhas secas de 50

TABELA 1 - Número de lesões de pinta preta em folhas de tomateiro tratadas ( $1^{\mathrm{a}}$ par de folhas) e não tratadas ( $2^{\mathrm{a}}$ par de folhas) com diferentes extratos brutos de plantas medicinais. Avaliação realizada 15 dias após a inoculação com Alternaria solani

\begin{tabular}{lcc}
\hline \hline Tratamentos & \multicolumn{2}{c}{ Número de lesões } \\
\cline { 2 - 3 } & $\begin{array}{c}\mathbf{1}^{\mathbf{0}} \text { par de } \\
\text { folhas }\end{array}$ & $\begin{array}{c}\mathbf{2}^{\mathbf{0}} \text { par de } \\
\text { folhas }\end{array}$ \\
\hline Testemunha (água) & $3,50 \mathrm{a}^{1}$ & $7,83 \mathrm{a}$ \\
A. millefolium $10 \%$ & $2,33 \mathrm{a}$ & $2,83 \mathrm{~b}$ \\
$20 \%$ & $2,50 \mathrm{a}$ & $3,00 \mathrm{~b}$ \\
R. officinalis $10 \%$ & $2,00 \mathrm{a}$ & $3,83 \mathrm{~b}$ \\
$20 \%$ & $1,33 \mathrm{a}$ & $3,67 \mathrm{~b}$ \\
C. citratus $10 \%$ & $2,00 \mathrm{a}$ & $2,17 \mathrm{~b}$ \\
$20 \%$ & $2,00 \mathrm{a}$ & $3,17 \mathrm{~b}$ \\
A. camphorata $10 \%$ & $2,16 \mathrm{a}$ & $2,00 \mathrm{~b}$ \\
$20 \%$ & $0,50 \mathrm{a}$ & $1,50 \mathrm{~b}$ \\
\hline
\end{tabular}

${ }^{1}$ Médias nas colunas, seguidas de mesma letra, não diferem entre si pelo teste de Scott-Knott, ao nível de 5\% probabilidade.

espécies de plantas reduziu o número de lesões da pinta-preta por planta. Resultados semelhantes foram observados por Balbi-Peña et al. (2006b), no qual a curcumina e os extratos de cúrcuma a $1 \%$ e $10 \%$ apresentaram níveis de controle de pinta preta causada por $A$. solani em tomateiro Bônus F1, similares ao tratamento com o fungicida oxicloreto de cobre, mas inferior ao fungicida azoxystrobin. A utilização de extratos de plantas medicinais está se tornando cada vez mais promissora no controle de doenças e deve ser testadas no campo. As plantas estudadas no presente trabalho podem se enquadrar neste contexto.

\section{REFERÊNCIAS BIBLIOGRÁFICAS}

Balbi-Peña MI, Becker A, Stangarlin JR, Franzener G, Lopes MC, Schwan-Estrada KRF (2006a) Controle de Alternaria solani em tomateiro por extratos de Curcuma longa e curcumina - I. avaliação in vitro Fitopatologia Brasileira 31:310-314.
Balbi-Peña MI, Becker A, Stangarlin JR, Franzener G, Lopes MC, Schwan-Estrada KRF (2006b) Controle de Alternaria solani em tomateiro por extratos de Curcuma longa e curcumina - II. Avaliação in vivo. Fitopatologia Brasileira 31:401-404.

Fiori ACG, Schwan-Estrada KRF, Stangarlin JR, Vida JB, Scapim CA, Cruz MES, Pascholati SF (2000) Antifungal activity of leaf extracts and essential oils of some medicinal plants against Didymella bryoniae. Journal of Phytopathology 148:483-487.

Franzener G, Stangarlin JR, Schwan-Estrada KRF, Cruz MES (2003) Atividade antifúngica e indução de resistência em trigo a Bipolaris sorokiniana por Artemisia camphorata. Acta Scientiarum 25:503-507.

Kuhn OJ, Portz RL, Stangarlin JR, Del Águila RM, Schwan-Estrada KRF, Franzener G (2006) Efeito do extrato aquoso de cúrcuma (Curcuma longa) em Xanthomonas axonopodis pv. manihotis. Semina: Ciência Agrária 27:13-20.

Kurozawa C, Pavan MA (2005) Doenças do tomateiro (Lycopersicon esculentum Mill.). In: Kimati H, Amorim L, Rezende JAM, Bergamim Filho A, Camargo LEA Manual de Fitopatologia: Doenças das plantas cultivadas, 4. ed. São Paulo SP. Ed. Agronômica Ceres, v. 2, pp. 607-626.

Montes-Belmont R, Garcia-Licona R (1997) Efecto de extractos vegetales en la germinacion de esporas y en los niveles de dano de Alternaria solani en tomate. Fitopatologia (Peru) 32:52-57.

Rodrigues E, Schwan-Estrada KRF, Fiori-Tutida ACG, Stangarlin JR, Cruz MES (2007) Atividade elicitora de fitoalexinas e proteção de alface em sistema de cultivo orgânico contra Sclerotinia sclerotiorum pelo extrato de gengibre. Summa Phytopathologica 33:124-128.

Rodrigues E, Schwan-Estrada KRF, Stangarlin JR, Cruz MES, Tutida-Fiori ACG (2006) Avaliação da atividade antifúngica de extratos de gengibre e eucalipto in vitro e em fibras de bananeira infectadas com Helminthosporium sp. Acta Scientiarum, Agronomy 28:123-127.

Schwan-Estrada KRF, Stangarlin JR (2005) Extratos e óleos essenciais de plantas medicinais na indução de resistência. In: Cavalcanti LS, Di Piero RM, Cia P, Pascholati SF, Resende MLV, Romeiro RS (Eds.) Indução de resistência em plantas a patógenos e insetos. PiracicabaSP. Fealq. pp. 125-132.

Stangarlin JR, Schwan-Estrada KRF Cruz MES, Nozaki MH (1999) Plantas medicinais e controle alternativo de fitopatógenos. Biotecnologia, Ciência \& Desenvolvimento 1:16-21.

Valarini PJ, Frighetto RTS, Melo IS (1994) Potencial da erva medicinal Cymbopogon citratus no controle de fitopatógenos do feijoeiro. Revista de Agricultura 69:139-150.

Vale FXR, Zambolim L, Paul PA, Costa H (2000) Doenças causadas por fungos em tomate. In: Zambolim L, Vale FXR, Costa H (Eds.) Controle de doenças de plantas - hortaliças. pp. 699-756.

Vicente MCM, Campbell A, Baptistella CSL, Coelho PJ, Lopes Júnior A (2002) O uso de agrotóxicos em culturas selecionadas da agricultura paulista. Informações Econômicas (São Paulo) 2:3444.

Recebido 16 Abril 2008 - Aceito 12 Junho 2008 - TPP 7133 Editor Associado Mário Lucio V. Resende 